\title{
Article
}

\section{Perceived facilitators to change in hospital pharmacy practice in England}

\author{
Auta, Asa, Maz, Julia and Strickland-Hodge, Barry \\ Available at http://clok.uclan.ac.uk/14189/
}

Auta, Asa ORCID: 0000-0001-6515-5802, Maz, Julia and Strickland-Hodge, Barry (2015) Perceived facilitators to change in hospital pharmacy practice in England. International Journal of Clinical Pharmacy, 37 (6). pp. 1068-1075. ISSN 2210-7711

It is advisable to refer to the publisher's version if you intend to cite from the work. http://dx.doi.org/10.1007/s11096-015-0153-9

For more information about UCLan's research in this area go to http://www.uclan.ac.uk/researchgroups/ and search for < name of research Group>.

For information about Research generally at UCLan please go to http://www.uclan.ac.uk/research/

All outputs in CLoK are protected by Intellectual Property Rights law, including Copyright law. Copyright, IPR and Moral Rights for the works on this site are retained by the individual authors and/or other copyright owners. Terms and conditions for use of this material are defined in the policies page.

\section{CLoK}

Central Lancashire online Knowledge www.clok.uclan.ac.uk

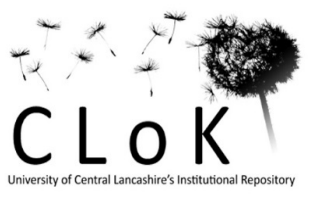




\section{PERCEIVED FACILITATORS TO HOSPITAL PHARMACY PRACTICE CHANGE IN ENGLAND}

Asa Auta,

School of Healthcare, Faculty of Medicine and Health, University of Leeds, Leeds, LS2 9JT, UK.

Julia Maz,

School of Healthcare, Faculty of Medicine and Health, University of Leeds, Leeds, LS2 9JT, UK.

Barry Strickland-Hodge,

School of Healthcare, Faculty of Medicine and Health, University of Leeds, Leeds, LS2 9JT, UK.

Corresponding author: Asa Auta, School of Healthcare, Faculty of Medicine and Health, University of Leeds, Leeds, LS2 9JT, UK. Email: $\underline{\text { hs09aa@leeds.ac.uk }}$ 


\section{Abstract}

Background: Traditionally, hospital pharmacists' roles have been associated with dispensing medications prescribed by doctors and offering advice about medicines to patients and other healthcare professionals. In England, significant changes in the structure of hospital pharmacy practice began in the 1970s and presently hospital pharmacists are undertaking a number of advanced roles including prescribing.

Objective: This study investigated the facilitators to change in hospital pharmacy practice in England in order to identify lessons that might assist in the potential changes needed in other countries for extended clinical roles.

Setting: The study was conducted in England

Methods: A qualitative study using semi-structured interviews was conducted with 28 participants, comprising 22 pharmacists and 6 pharmacy technicians from England. They were recruited through a snow ball sampling technique. Transcribed interviews were entered into the QSR NVivo 10 software for data management and analysed thematically.

Main outcome measure: Pharmacists and pharmacy technicians' perception of the facilitator to hospital pharmacy practice change in England.

Result: Three major themes emerged from this study: drivers for change, strategies for change and efficiency. Many of the drivers identified were linked to changes in the structure of hospital pharmacy including education and training; specialisation in practice and career structure. Strategies employed to achieve practice change included broadening of the role of pharmacy technicians in order to free-up pharmacists' time; seizing opportunities for extended roles; developing a relationship with the medical profession, and professional leadership influence. Participants perceived that the development of pharmacists' clinical roles have resulted in a more efficient healthcare provision where patients were offered seamless services.

Conclusion: Changes in the professional structure of pharmacy including education and training, specialisation, career structure and the roles of pharmacy technicians could benefit the development of pharmacists' clinical roles in other countries.

Key words: Hospital pharmacy, role development, pharmacy practice change, facilitators, England 


\section{Impact of findings on practice}

- Changes in the structure of hospital pharmacy could benefit the development of advanced clinical pharmacy practitioners in many countries.

- A clinical career pathway has the potential to make pharmacists aspirational and motivate them to develop clinical competencies that are needed for advanced practice.

- Specialisation in practice is essential to enhance pharmacists' contribution to patient care.

- Appropriate use of pharmacy technicians could free-up pharmacists to do more clinical work.

\section{Introduction}

Traditionally, hospital pharmacists' roles have been associated with dispensing medications prescribed by doctors and offering advice about medicines to patients and other healthcare professionals. However, in some countries including the United States of America (USA) and United Kingdom (UK), their roles have been expanded in order to optimise patient care and the utilisation of pharmacists' skills [1].

Pharmacists are highly educated professionals whose expertise in medicines management have been underutilised in many countries [1]. In most European countries, pharmacists' basic education consists of a 4-year masters level undergraduate degree with a significant clinical component [2]. Even though there are diversities in pharmacy education in Africa and Asia, many institutions in these continents run Bachelor of Pharmacy programmes which are similar to the training model in Europe. A number of institutions also run the Doctor of Pharmacy programme (Pharm D.) which has extended clinical components [3, 4]. Therefore, advances in pharmacy education and medical practice require that pharmacists who are experts in medicines management take an active role in patient care.

In England, hospital pharmacists have expanded their roles beyond the dispensary, initially with the introduction of ward pharmacy and medicines information which were followed by the provision of clinical pharmacy services including reviewing patients' medications, monitoring patients' therapy and advising doctors on drug therapy [5]. Today, hospital pharmacists are directly involved in patient care working as specialists among healthcare teams in different clinical areas. In addition, some act as prescribers 
of medicines either as independent (autonomous) or supplementary prescribers (working within a clinical management plan following diagnosis by an 'independent' medical or dental practitioner) [6].

There is theoretical evidence to suggest that the implementation of the 1970 report (Noel Hall report) of the working committee on the Hospital Pharmaceutical Service in Great Britain was a major facilitator for change in hospital pharmacy practice $[7,8]$. This report stated that hospital pharmacists can no longer be regarded only as dispensers of medicines. Therefore, they have a role in ensuring safe and cost effective use of medicines on the wards [9]. Also, other literature seem to view the implementation of the recommendations of the committee of enquiry appointed by the Nuffield foundation in 1983 as the bedrock for the current pharmacy practice [10, 11]. This was because the Nuffield report suggested that clinical pharmacy practice should be established in all hospitals [12]. This led to a number of reorganisations in the structure of hospital pharmacy including changes in pharmacists' training and the development of specialist clinical roles. Furthermore, recent reports suggest that the pharmacists' current role in prescribing is supported by the changes that occurred in the professional structure of hospital pharmacy practice prior to the introduction of pharmacist prescribing $[13,14]$. For example, pharmacist supplementary prescribing was easily adopted in clinical areas where hospital pharmacists had existing specialist roles $[13,14]$.

However, empirical research exploring pharmacy staff views on the changes in the structure of hospital pharmacy practice that have enabled and supported pharmacists' clinical roles and the strategies employed to achieve change is lacking. Therefore, we explored pharmacy staff opinion on the facilitators to changes in hospital pharmacy practice in England as part of a larger qualitative study. The larger study aimed at investigating the changes that occurred in the structure of pharmacy practice prior to the introduction of pharmacist prescribing in the UK. This paper draws from the data obtained from participants in England only.

\section{Aim of the study}

To investigate the facilitators to changes in hospital pharmacy practice in England in order to identify lessons that might assist in the potential changes needed in other countries for extended clinical roles. 


\section{Ethics approval}

Ethical approval for this study was obtained from the School of Healthcare Research Ethics Committee (Ref no: SHREC/RP/344). All participants were provided with an information sheet containing details of the research and written, informed consent was obtained from them.

\section{Methods}

\section{Study design}

This research employed a qualitative study design using semi-structure interviews in order to gain an in-depth understanding of the changes that occurred in the structure of pharmacy practice prior to the introduction of pharmacist prescribing in the UK. This paper utilises the data obtained from participants in England.

\section{Settings, recruitment and sampling strategy}

We recruited pharmacists and pharmacy technicians in England who qualified from 1970 to 1999. The rationale for this was because significant changes in pharmacy practice in England occurred within this period. These pharmacy staff would be able to report their experiences of pharmacy practice and the changes they have seen since qualifying.

A convenience sample of pharmacists who were part of the Royal Pharmaceutical Society virtual network of Prescribing and Medicines Management $(n=312)$ plus those who attended the prescribing course at the University of Leeds $(n=30)$ were sent a generic email in October 2013. In the mail, we informed prospective participants that we intend to recruit pharmacists and pharmacy technicians who qualified from the 1970 to 1999 in our research that is looking at role development in hospital pharmacy. Those that indicated an interest to participate and who met the inclusion criteria were invited for an interview. Participants who were outside the West Yorkshire region of England were interviewed by telephone.

Seven (7) participants in England were recruited from the initial contact made. A snowball sampling strategy was employed to recruit other participants. In this strategy, participants who had been interviewed were asked if they knew other pharmacy colleagues who may be relevant to this research and if they were willing to forward the generic email they received to those colleagues. Those who received the forwarded 
email contacted the researchers directly if they were interested in participating in the study. This referral system continued until data saturation was achieved. This snowball strategy was effective as all remaining participants were recruited through this approach.

\section{Data collection}

Semi-structured, in-depth interviews were conducted from October 2013 to February 2014. The interview guide was based on a review of the literature. Topic areas covered included the changes in pharmacy practice experienced by participants; facilitators and barriers to implementing these changes; advantages and disadvantages associated with changes and lessons that can be learned by other countries where clinical pharmacy practice is still underdeveloped. The interview guide was reviewed by a panel of 3 researchers for content validity. The interview guide was pilot tested among two participants, a pharmacist and a pharmacy technician. The data obtained from the pilot study were included in the overall results of the study as there were no major changes to the content and structure of the interview guide after the pilot.

All interviews were conducted by AA. The interviews conducted in this study were continued until the researcher perceived that data saturation was reached i.e. no new data was generated. In all, 28 interviews were conducted with participants in England and they lasted between 30-80 minutes.

\section{Data analysis}

The interviews conducted were digitally recorded and fully transcribed. AA checked each transcript against the digital audio recording for accuracy. Transcript data were entered into the QSR NVivo 10 software for appropriate data management. Data were analysed using thematic analysis in the manner outlined by Braun and Clarke [15]. The analysis proceeded in iterative stages of data familiarisation, generation of codes, searching, reviewing, defining and naming of themes. The data were independently coded and analysed by one researcher (AA) but reviewed at different stages by two additional researchers including reviewing and refining the coding structure and identified themes. At the final stage of the analysis, identified themes were discussed by the three researchers until a consensus was reached. Respondent validation was carried out by asking two of the participants interviewed to comment on the appropriateness of the interpretation of the findings and provide feedback. These 
respondents were satisfied with the data analysis and interpretation made. However, they provided a few comments which were considered in the final interpretation of the data.

\section{Results}

Of the 28 interviews conducted, 13 were face-to-face and 15 were via the telephone. The participants were 22 pharmacists and 6 pharmacy technicians. Fourteen (14) of these participants qualified in the 1970s; 10 qualified in the 1980s and 4 qualified in the 1990s. Participants' current practice settings included hospitals (12), primary care (6), academics (6), leadership (1) and the remaining 3 were retired. All those who participated in the study had worked in hospital pharmacy at some point during the 1970 - 1999. However, 3 of them left hospital practice at a junior grade. Seven (7) of the participants had worked in a community pharmacy either in a full-time or part time position. Hospital pharmacists interviewed included directors/chief pharmacists (4), service managers (4) and a consultant pharmacist. Seven (7) of the hospital pharmacists interviewed had prescribing as part of their roles.

Although, this study was intended to cover developments in hospital pharmacy practice prior to the introduction of pharmacist prescribing, respondents made comments about other relevant recent developments which were included in this report. Overall, three major themes emerged from this study: drivers and barriers; strategies for change and efficiency. These themes are represented in Figure 1 using the Input, Process, Output model to show their relationships.

[Insert figure here]

\section{Drivers}

This theme relates to the factors that enabled hospital pharmacists in England to develop their clinical roles. Participants reported that there were drivers from the government that resulted in significant changes in hospital pharmacy. They commented on several government documents and legislation from the Noel Hall report of 1970 to the Health and Social Care Act of 2001 that enabled pharmacists to become prescribers. However, many participants saw the implementation of the Noel Hall report as an initial key driver because it provided a better career structure for 
hospital pharmacists and promoted the recruitment and retention of experienced clinical staff.

"As at the time I joined hospital pharmacy there were certainly difficulties with recruitment and the career structure was very limited. So, people looking for long term career were not necessarily looking to hospital pharmacy." (P11, Pharmacist)

"The Noel Hall report was sort of a kick off to getting the things going." (P17, Pharmacist)

Participants also reported on the changes in education and training of pharmacists. These changes including the introduction of the postgraduate clinical pharmacy programmes were made to address many of the shortcomings of pharmacists' clinical knowledge and skills as a result of the expansion of pharmacy services into clinical areas. Many participants perceived that these clinical programmes have enabled hospital pharmacists to develop more depth in their clinical knowledge and become more confident in performing clinical roles.

“...those diplomas have been an important part of arming pharmacists with the clinical abilities..." (P07, Pharmacist)

The medical profession was also acknowledged by participants as pharmacy practice change agent. Many participants mentioned that there was resistance from their medical colleagues in the early days of clinical pharmacy as many doctors perceived it to be an encroachment into their roles. However, participants noted that medical doctors were instrumental in bringing about change as they were involved in the training of the initial cohorts of clinical pharmacists.

"I undertook a masters' in clinical pharmacy and this was brand new... Initially we were taught by medical staff." (P24, Pharmacist)

Many participants also saw the opportunity to specialise in practice and the recent introduction of the consultant pharmacist post in 2005, which provided a clinical career pathway for hospital pharmacists, as significant drivers to retention of experienced pharmacists in clinical roles. 
“....the career structure now reflects a very clear opportunity to specialise and sub-specialise at the unit here, whereas at one time, you know you are in a position where it sort of led into the management route really as opposed to specialised into clinical level" (P04, Pharmacist)

However, some pharmacists interviewed expressed concern over the present consultant pharmacist appointment model as it does not provide as many specialist clinical pharmacists the opportunity to get to the post.

"I think in my own hospital I have a large number of pharmacists who would be capable of practising as pharmacist consultants but we don't have the post available for them to go into." (P15, Pharmacist)

This study revealed that major obstacle to increasing the current number of consultant pharmacist posts were around funding.

Another factor that drove change was the increasing positive attitude of pharmacists towards clinical roles. Participants felt that this came about as a result of the change in pharmacy education. In an interview with a pharmacist who graduated in the 1990s, he exclaimed:

"I didn't do a pharmacy degree to do the counting tablet role! I did it because I wanted to do clinical pharmacy, I wanted to be on the ward, I wanted to be influencing doctors prescribing, I wanted to be counselling patients on their medicines, I wanted to make sure that the medicines were being used in a safe way providing my expertise" (P18, Pharmacist)

This dissatisfaction with medicine supply roles was commonly reported as a significant driver for the adoption of new clinical roles by younger pharmacists.

\section{Strategies for change}

Based on participants' reports, a number of strategies were identified to have facilitated change. These included freeing-up pharmacists' time for clinical roles by shifting some of the pharmacists' traditional supply roles to pharmacy technicians. Participants' accounts revealed that in the 1970s and 1980s, the shortage of hospital pharmacists was a major barrier to the development of clinical pharmacy services. 
However, the development of pharmacy technicians, to take on roles previously undertaking by pharmacists including accuracy check enabled hospital pharmacists to undertake more clinical roles on the ward.

"The next biggest change that I saw was the change to technicians starting to do accuracy check and I think that was a huge step..." (P03, Pharmacy Technician)

"In the hospital, the technicians freed up pharmacists time to actually be more visible on the ward and have more impact in terms of prescribing advice especially with the junior doctors." (P06, Pharmacist)

Despite this development, many pharmacists observed that the post-registration training of technicians needed to be well coordinated. These participants suggested the necessity of establishing a national framework due to the increasing level of technicians' involvement in medicines management roles and the reliance of pharmacists on them.

“...there isn't anything formal in the country, everybody does it locally.” (P29, Pharmacist)

"Post registration training is done locally. I think we probably need to get some standardisation into that..." (P15, Pharmacist)

Some participants also reported seizing the opportunity for role expansion by identifying gaps in clinical practice that could be filled by pharmacists. A pharmacist interviewed narrated how they took advantage of the national strike action by other hospital staff to establish one of the first pharmacist-led specialist clinics in the UK.

"So, XXX [pharmacist's name] and I said to the medical consultant, "well, we can help you with that, we can help you look at some of the patients for you". So we had patients coming to us for [treatment] where we have to adjust the doses of [medication] and established what was in effect one of the first pharmacist-led [specialist] clinic in the country." (P32, Pharmacist)

Furthermore, participants described key individuals using various terms including 'leading individuals', 'forward thinking pharmacists', 'role models' and 'champions of 
change' who had different perspectives of pharmacists' professional roles. These key individuals were either chief pharmacists or other principal pharmacy staff who had clear vision of pharmacists doing more of clinical and less of dispensary-based work. These individuals were able to communicate this vision to other pharmacy staff and to facilitate it happening locally.

"XXX hospital has been at the forefront of clinical pharmacy ever since it started and that was because of one or two chief pharmacists who pushed it forward. So it was individuals really who made the difference." (P07, Pharmacist)

Similarly, participants commonly mentioned engaging the United Kingdom Clinical Pharmacy Association (UKCPA) to campaign for change at the national level. Some hospital pharmacists noted that they had to engage UKCPA rather than the Royal Pharmaceutical Society of Great Britain to push for change centrally because they felt the society was not proactive toward extended clinical roles for hospital pharmacists at that time

"We had to use UKCPA to do that because at that time the Pharmaceutical Society was not engaged with that at all, they were more engaged with other aspect of the profession and not in clinical practice, they only came to recognise clinical practice much later on." (P32, Pharmacist)

However, these participants acknowledged that this is no longer the case.

Also, developing a relationship with the medical doctors was another strategy employed by hospital pharmacists. A pharmacist who was newly employed in a hospital in the early days of clinical pharmacy reported how he was able to do clinical work on the ward as a result of the rapport his chief pharmacists had with medical staff.

"...XXX who was the chief pharmacist there [name of a hospital] had excellent working relationships with the medical staff in the hospital and so I routinely spent most of my week working clinically with medical consultants..." (P32, Pharmacist) 
Many pharmacists felt that the initial medical consultants who they work with, who saw the benefits of the pharmacists being on the wards were instrumental in recommending them to their other colleagues.

\section{Efficiency}

Many participants perceived that the development of hospital pharmacists' roles have resulted in a more efficient healthcare provision. Participants who were prescribers mentioned that taking on that extended role contributed to the seamless services experienced by patients. They talked about how patients now need not wait for doctors to adjust doses or add medicines which were missing on their prescriptions in areas where you have a pharmacist prescriber.

"It means that they are now getting their medicine promptly and in a correct manner and one would hope less potential for error." (P23, Pharmacist).

Pharmacists now view themselves as an indispensable part of the healthcare team and considered their services as essential.

"If I think about where we are now, if pharmacists stop doing what they are doing on the ward, then the system would fall down completely but in those days, if we stopped what we were doing on the ward, things would have probably kept going okay." (P07, Pharmacist).

Participants also noted other benefits resulting from taking on extended clinical roles including enhanced relationships with other members of the healthcare team; better use of pharmacists' skills; and increase in job satisfaction for pharmacy staff.

"I would have been fed up if I spent a lot of time counting or being in a dispensary, that just wouldn't suit me. So, I feel personally more satisfied in my job because I have got this ability to work clinically." (P27, Pharmacist).

Other economic benefits including effective management of medicine budgets through pharmacists' involvement in formulary management and advice offered to medical prescribers on cost-effective treatments; and cost effective use of the skill-mix within pharmacy were also reported. 


\section{Discussion}

This is the first study that primarily investigated changes in the structure of hospital pharmacy practice and link that to the development of pharmacists' clinical roles in England. Many of the drivers identified including government policies, enhanced education, task shifting to pharmacists' support staff, relationship with medical doctors and leadership are fairly known and have been linked to the development of clinical pharmacy practice in hospitals $[16,17]$. For example, Penm et al. [17] investigated the development of clinical pharmacy practice in China by interviewing 130 participants from 29 hospitals. They reported that government support, adequate pharmacy staffing and clinical pharmacy training were crucial for the development of clinical pharmacy practice in hospitals in China. Furthermore, a cross-sectional survey involving 726 hospital pharmacy directors in 31 countries of the Western Pacific Region identified similar facilitators [16]. This study reported that individual pharmacist trait, hospital pharmacy resources including staffing, government and physician support were key facilitators to clinical pharmacy services in this region [16]. However, our findings adds to the international literature by suggesting that within hospital pharmacy practice, a clinical career pathway that allows progression within clinical specialty could sustain and support the development of pharmacists' clinical roles. This is because a clinical career route has the potential to make pharmacists aspirational and motivate them to develop clinical competencies that are needed for advanced practice in order to progress through the career ladder. In addition, it also promotes the retention of experienced clinical staff who desire to use their expertise to enhance patient care rather than move into managerial roles where their clinical expertise would be untapped.

The present study also suggests that specialisation is supported by a clinical career route. Specialisation as opposed to generalist practice is essential to enhance pharmacists' contribution to patient care considering that pharmacists will have to work with other specialists in multidisciplinary teams and the complex nature of disease and medicines. Although studies comparing the relative impact of specialist and nonspecialist pharmacists in patient care are lacking, a systematic review conducted to assess the impact of care provided to cancer patients by specialists and non-specialist clinicians showed that the patients receiving specialists' care had better health outcomes including reduced mortality rate than those receiving care from non- 
specialists [18]. Pharmacists' specialisation is likely to have a similar impact on patient care. This is because a few studies including Leape et al. [19] have revealed that pharmacists' contribution in patient care including drug therapy interventions is enhanced when an experienced pharmacist specifically works with a specialist team as opposed to a generalist.

As highlighted in the results section, the development of credible practitioners is essential in achieving clinical change in pharmacy practice. This depends on the robustness of training received by pharmacists. Pharmacy training particularly at undergraduate level is evolving to meet the challenges of healthcare provision in hospital and community pharmacy settings. Even in countries where pharmacists' roles have been expanded considerably, the undergraduate curriculum is constantly being reviewed in order to prepare pharmacists for future roles [20]. For example, in the UK, the undergraduate pharmacy curriculum has been restructured to include more clinical components and practice-based learning. Procedures are on the way to integrate more fully the pre-registration year into the undergraduate programme [20]. The reasoning behind this is to produce more competent pharmacists able to apply their knowledge and skills in clinical settings upon graduation. In Canada, there are transitional arrangements to replace, by 2020 , the existing baccalaureate in pharmacy with doctor of pharmacy programmes which has a more experiential learning component in clinical settings [21]. Furthermore, post-registration clinical training is increasingly becoming a pre-requisite for advanced clinical practice in hospitals in many countries, including the UK, Portugal and Spain [2]. These educational developments are necessary in order to meet the challenges of expanded clinical roles.

Major barriers to the extension of pharmacists' clinical roles in many countries including some developed countries, are pharmacists' shortages and lack of time to take on extended roles $[22,23]$. For example, a review of pharmacy practice in rural Queensland, Australia revealed that extended clinical pharmacy services have been hindered by a shortage of pharmacists [23]. Evidence from this study also demonstrated that appropriate use of pharmacy technicians could free up pharmacists to do clinical work. Other research evidence has shown that suitably trained pharmacy technicians could handle some medicine management roles, including medicine history taking $[24,25]$. Therefore, adequately trained pharmacy technicians or any 
other equivalently trained pharmacists' support staff that are available in other countries, are essential for developing clinical pharmacy practice.

Overall, a key factor driving change in hospital pharmacy practice in England is the government's support through appropriate policies and legislation. Therefore, in other countries considering change, government support is essential because some extended clinical roles will require relevant legislation or policy change. For example, legislation would be needed to enable hospital pharmacists working within multidisciplinary health teams to modify patients' therapy including adjusting dosages or duration of therapy; or for community pharmacists to supply some prescribed medications under emergency conditions. A number of factors drive government support for extended clinical roles including improving access to care, utilisation of pharmacists' skills, cost saving on medicines budgets and cost effective use of available human resources [26]. Therefore these drivers could be used by pharmacists in countries where pharmacists' roles are limited, to advocate for government support for extended roles.

Furthermore, the traditional structure of hospital pharmacy practice in many countries particularly in Asia and Africa, is characterised by limited roles with dispensing medicines as the core function of pharmacists; a generalist approach to practice; limited post-registration education and training to enhance pharmacists skills; limited roles for pharmacy technicians; a single career pathway that is increasingly managerial as pharmacists progress through their career; and professional isolation of pharmacists from other members of the healthcare team [1, 27]. The evidence from this study suggests that a change in this traditional structure could benefit the development of highly specialised clinical pharmacists working at advanced levels to optimise patient therapy in these countries. However, this should be treated with caution as developing countries differ significantly from England in the structure and organisation of their health systems including pharmacy practice. Therefore, further research evidence particularly in developing countries is needed in order to confirm the claim of this study. Thus, we extended the current study to investigate the structure of hospital pharmacy practice in Nigeria in order to determine what structures would support the development of pharmacists' clinical roles in a developing country. 
Finally, certain limitations were associated with this study. First, being a qualitative study, the findings cannot be statistically generalised. Notwithstanding, certain concepts from the findings of this study could be transferable to other context. For example, the development of pharmacy technicians or other category of pharmacists' support staff, to do some of the traditional roles of pharmacists could be crucial in freeing up pharmacists time to do extended clinical roles in many countries. Secondly, it is possible that participants in this study had difficulties recalling events that happened in the earlier years of their practice. However, this was not explicitly shown. Furthermore, many of the participants interviewed showed a lack of understanding of the structure of pharmacy practice in countries where clinical pharmacy practice is still underdeveloped. Therefore, they were unable to comment on what lessons that could be learned by these countries. Despite these limitations, the key findings of the current study would be of interest to many countries considering change in hospital pharmacy practice.

\section{Conclusion}

Hospital pharmacists in the England have successfully redefined their roles and are now working at advanced levels clinically. Their current clinical roles have been supported by changes in the professional structure of pharmacy including a shift in the focus of pharmacy training from purely scientific to a patient oriented one; specialisation in practice; a career route that recognised advanced pharmacy practitioners; enhanced working relationships with doctors and the development of pharmacy technicians to do some roles previously undertaken by pharmacists. This study suggests that changes in the structure of pharmacy practice could benefit the development of hospital pharmacists' clinical roles in many countries where pharmacists' roles are limited. Finally, this is a national study, carried out predominantly in England and whilst the findings may be of interest to an international audience, the conclusions may not be applicable to all settings.

\section{Acknowledgement}

The authors are grateful to all the participants of this study. 


\section{Funding}

No financial support was obtained for this study.

\section{Conflict of interest}

The authors do not have any conflict of interest to declare.

\section{Reference}

1. Doloresco F, Vermeulen LC. Global survey of hospital pharmacy practice. Am J Health Syst Pharm. 2009;66(5 Supplement 3):s13-s19.

2. Van Mil J, Schulz M. A review of pharmaceutical care in community pharmacy in Europe. Harvard Health Policy Rev. 2006;7(1):155-168.

3. A lo A. Pharmacy in Nigeria. Am J Health Syst Pharm. 2006;63:670-673.

4. Basak SC. Sathyanarayana D. Pharmacy education in India. Am J Pharm Educ. 2010;74(4):68.

5. Child D, Cooke J. Clinical pharmacy services. In: Hospital Pharmacy, Stephens M, ed. London: Pharmaceutical Press, 2003. ISBN 0853695024.

6. Stewart D, MacLure K, George J. Educating nonmedical prescribers. Br J Clin Pharmacol. 2012;74(4):662-667.

7. Anderson S. Making medicines: a brief history of pharmacy and pharmaceuticals. London: Pharmaceutical Press, 2005. ISBN 0853695970.

8. Kay EA. The changing role of the clinical pharmacist in rheumatology. Rheumatology. 1986;25(1):87-90.

9. Report of the working party of the hospital pharmaceutical service. London: Department of Health and Social Security Scottish Home and Health Department Welsh Office, 1970. SBN 113209940.

10. Anderson S. The historical context of pharmacy. In: Pharmacy practice, Taylor K and Harding G, eds. London: Taylor \& Francis, 2001. ISBN 0203303156.

11. Silcock J, Raynor DKT, Petty D. The organisation and development of primary care pharmacy in the United Kingdom. Health Policy. 2004;67(2):207-214.

12. The report of a committee of inquiry appointed by the Nuffield Foundation. 1986, London: The Nuffield Foundation, 1986. ISBN 0904956245.

13. Hobson RJ, Sewell GJ. Supplementary prescribing by pharmacists in England. Am J Health Syst Pharm. 2006;63(3):244-253.

14. Dawoud D, Griffiths P, Maben J, Goodyer L, Greene R. Pharmacist supplementary prescribing: a step toward more independence? Res Social Adm Pharm. 2011;7(3):246-256.

15. Braun V, Clarke V. Using thematic analysis in psychology. Qual Res Psychol. 2006;3(2):77-101.

16. Penm J, Chaar B, Moles R. Clinical pharmacy services that influence prescribing in the Western Pacific Region based on the FIP Basel Statements. Int J Clin Pharm. 2015;37(3): 485-496. 
17. Penm J, Moles R, Wang H, Li Y, Chaar B. Factors affecting the implementation of clinical pharmacy services in China. Qual Health Res. 2014;24(3):345-356.

18. Grilli R, Minozzi S, Tinazzi A, Labianca R, Sheldon TA, Liberati A. Do specialists do it better? The impact of specialization on the processes and outcomes of care for cancer patients. Ann Oncol. 1998;9(4):365-374.

19. Leape LL, Cullen DJ, Clapp MD, Burdick E, Demonaco HJ, Erickson JI, et al., Pharmacist participation on physician rounds and adverse drug events in the intensive care unit. JAMA.1999; 282(3):267-270.

20. Smith A, Darracott R. Review of pharmacist undergraduate education and preregistration training and proposals for reform: Discussion paper. 2011, Modernising Pharmacy Careers Programme: Medical Education England. http://hee.nhs.uk/healtheducationengland/files/2012/10/Pharmacist-preregistration-training-proposals-for-reform.pdf. Accesed 11 October 2014.

21. Canadian Pharmacists Association. CPhA position statement on a Doctor of Pharmacy degree as an entry-level to practice. 2011. http://www.pharmacists.ca/cpha-ca/assets/File/cpha-on-theissues/PPDoctorOfPharmacyEN.pdf. Accessed 12 June 2014.

22. International Pharmaceutical Federation. FIP global pharmacy workforce report. The Hague: Fédération Internationale Pharmaceutique (FIP), 2012. http://www.fip.org/files/members/library/FIP_workforce_Report_2012.pdf. Accessed 13 July 2014.

23. Tan ACW, Emmerton L, Hattingh HL. A review of the medication pathway in rural Queensland, Australia. Int J Pharm Pract. 2012;20(5):324-339.

24. Johnston R, Saulnier L, and Gould O. Best possible medication history in the emergency department: comparing pharmacy technicians and pharmacists. Can J Hosp Pharm. 2010;63(5):359.

25. Michels RD, Meisel SB. Program using pharmacy technicians to obtain medication histories. American journal of health-system pharmacy: Am J Health Syst Pharm. 2003;60(19):1982-1986.

26. Review of prescribing, supply \& administration of medicines. Final report. 1999.

http://webarchive.nationalarchives.gov.uk/20130107105354/http:/www.dh.gov. uk/prod_consum_dh/groups/dh_digitalassets/@dh/@en/documents/digitalass et/dh_4077153.pdf. Accessed 23 May 2013.

27. Anderson S. The state of the world's pharmacy: a portrait of the pharmacy profession. J Interprof Care. 2002;16(4):391-404. 
Figure
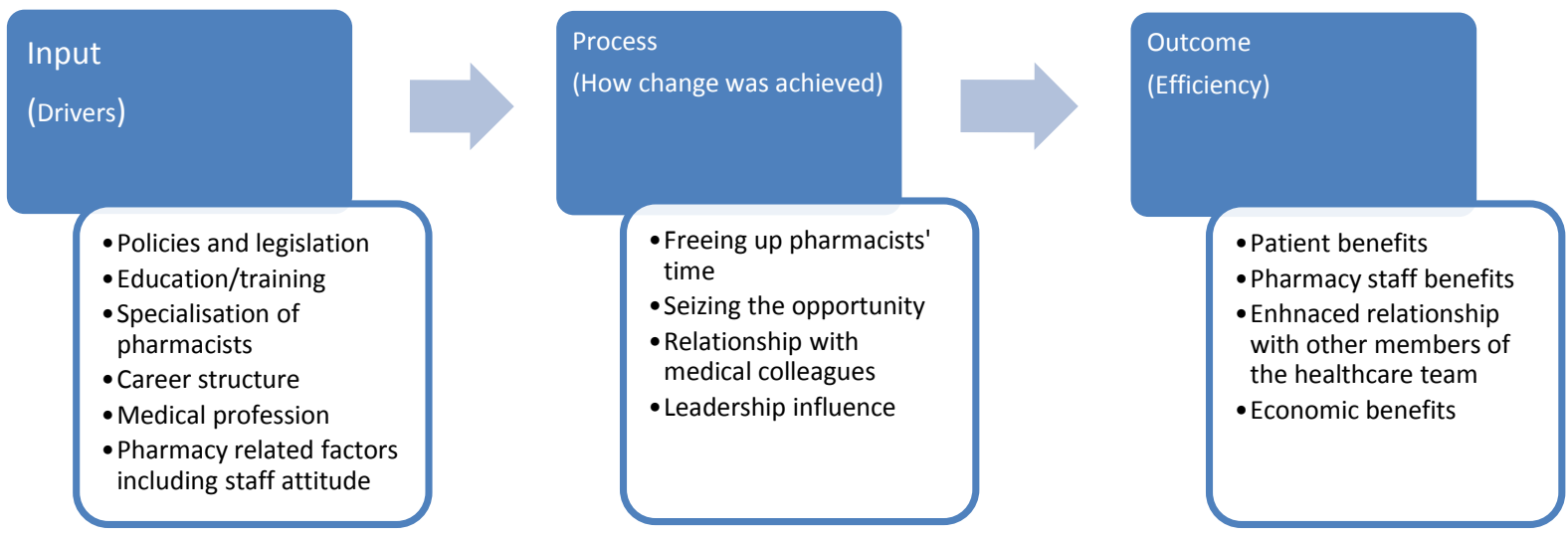

Figure 1: An overview of themes identified and their relationships. 